\title{
Does cryptogenic fibrosing alveolitis carry an increased risk of death from lung cancer?
}

\author{
J M Harris, P Cullinan, J C McDonald
}

It is widely believed among chest physicians that patients with cryptogenic fibrosing alveolitis (CFA) are at substantially increased risk of death from lung cancer. This belief is largely the result of a follow up study by TurnerWarwick and colleagues reported in $1980 .{ }^{1}$ Of 205 patients attending the Brompton Hospital between 1955 and 1973 with CFA, 20 (9.8\%) died from lung cancer; an excess relative risk of 14.1. This association is often taken to suggest that pulmonary fibrosis in itself entails a mechanism that predisposes to malignancy and that this in turn may explain the observed risk between other fibrotic diseases such as asbestosis and silicosis and lung cancer. The scientific importance of the question, and its medicolegal implications, led us to seek confirmatory evidence of the CFA cancer relation beyond the confines of a single highly specialised hospital. As a first step we have compared the frequency with which lung cancer is mentioned on death certificates of persons dying from CFA and other fibrotic lung diseases.

\section{Method}

All deaths in England and Wales are registered by the Office for National Statistics (ONS). Death certificates comprise two sections; the cause leading directly to death and causes antecedent to it are listed in Part I, any other causes contributing to death are listed in Part II. The lowermost cause of death in Part I is termed the "underlying cause", all other causes listed are "mentioned causes". Coded mentioned causes of death are only available for 1985 and 1986.

We obtained from ONS information on all certificates within this period that mentioned at least one of four fibrotic diseases of interest: asbestosis (code 501, ICD IX), pneumoconiosis resulting from other silica or silicates (ICD 502), coalworkers' pneumoconiosis (CWP, ICD 500); and other alveolar and parietoalveo- lar pneumopathy (ICD code 516). Idiopathic (or cryptogenic) fibrosing alveolitis (ICD 516.3) accounted for $91 \%$ of deaths where ICD 516 was coded. Within each of the four groups we compared the frequencies with which malignant neoplasm of trachea, bronchus, and lung (ICD 162) was also recorded.

\section{Results}

CFA was recorded on 1951 death certificates, asbestosis on 418, silicosis on 176, and CWP on 1345 . The sex and age distributions of these certificates are shown in table 1; those who died with CFA or asbestosis were slightly younger than those who died with silicosis or CWP. In $43 \%$ of certificates with asbestosis recorded, lung cancer was also mentioned; however, the proportions were consistently lower for certificates with CFA (6\%), silicosis $(7 \%)$ or CWP $(8 \%)$ recorded. These differences remained after stratification by sex and in those aged more or less than 75 years, the approximate median age for all the deaths.

\section{Discussion}

Among the certificates analysed, the frequency with which lung cancer and CFA were both recorded was no different than for silicosis or CWP and much lower than for asbestosisfindings unexplained by differences in age or sex. These occupational pneumoconioses were chosen for comparison because of their known association, or lack of it, with lung cancer and also because such patients would have been subject to similar levels of radiographic investigation. The association of asbestosis and silicosis with lung cancer is well known, and no such relation has been found with CWP. ${ }^{2}$

There is evidence that inaccuracy in death certification in Britain underestimates deaths resulting from CFA by as much as $60 \%{ }^{3}$ The omission from death certificates of pulmonary fibrosis in patients dying with lung cancer may

Accepted for publication 24 April 1998

Table 1 Proportion of death certificates on which lung cancer was recorded in addition to CFA, asbestosis, silicosis or CWP (England and Wales 1985/6)

\begin{tabular}{|c|c|c|c|c|c|c|c|c|c|c|}
\hline \multirow[b]{2}{*}{ Fibrotic disease ${ }^{\star}$} & \multirow{2}{*}{$\begin{array}{l}\text { Number of } \\
\text { certificates }\end{array}$} & \multirow[b]{2}{*}{$n(\%)$ male } & \multicolumn{3}{|c|}{ Mean age $(y)(S D)$} & \multicolumn{5}{|c|}{$n(\%)$ lung cancer also mentioned } \\
\hline & & & all & male & female & all & male & female & $<75$ years & $\geqslant 75$ years \\
\hline CFA (ICD 516) & 1951 & $1227(63)$ & $68(17)$ & $67(17)$ & $69(19)$ & $109(6)$ & $93(8)$ & $16(2)$ & $80(7)$ & $29(4)$ \\
\hline Asbestosis (ICD 501) & 418 & $375(90)$ & $67(9)$ & $66(9)$ & $70(9)$ & $180(43)$ & $168(45)$ & $12(28)$ & $152(46)$ & $28(31)$ \\
\hline Silicosis (ICD 502) & 176 & $164(93)$ & $74(8)$ & $74(8)$ & $81(5)$ & $14(8)$ & $14(9)$ & 0 & $4(5)$ & $10(10)$ \\
\hline CWP (ICD 500) & 1345 & $1340(100)$ & $75(7)$ & $75(7)$ & $71(15)$ & $98(7)$ & $98(7)$ & 0 & $48(9)$ & $50(6)$ \\
\hline
\end{tabular}

^As either underlying cause or other disease also present. 
be influenced by the type of fibrosis or by the certifying doctor's views about the existence or importance of the association. Thus while it seems likely that patients with well established asbestosis, who later die of lung cancer, will have both diseases recorded on the death certificate, this may be less probable for silicosis and even less for CWP. Conversely, the affirmed link between CFA and lung cancer is well known by chest physicians by whom most cases are likely to have been seen at some stage.

The survey by Turner-Warwick et al has its uncertainties. In case series of this kind, comprising patients already gravely ill and with very high short-term mortality, it is difficult to imagine an appropriate basis for comparison. Certainly the mortality experience of the general population of England and Wales is unsuitable. Secondly, patients were selected "in whom external fibrotic agents could be excluded". It seems doubtful whether in 1955-1973 sufficient was known about occupational asbestos exposure to have reliably removed this cause of pulmonary fibrosis and associated lung cancer.

We conclude that neither the study by Turner-Warwick et al nor our own can give an unequivocal answer to the question of whether patients with CFA are at increased risk of death from lung cancer. This is an important question with wide-ranging implications that calls for further well controlled study.

1 Turner-Warwick M, Lebowitz M, Burrows B, Johnson A. Cryptogenic fibrosing alveolitis and lung cancer. Thorax 1980;35:496-9.

2 IARC. Evaluation of the carcinogenic risk of chemicals to humans (silica and some silicates). IARC Monographs volume 68. Lyon: IARC, 1997:392.

3 Johnston I, Britton J, Kinnear W, Logan R. Rising mortality from cryptogenic fibrosing alveolitis. BMF 1990;301:101721 